\title{
Higher order modes and topological phase in the coiled elliptical weakly guiding optical fibres
}

\author{
Alexeyev C.N., Lapin B.P. and Yavorsky M.A. \\ Taurida National V.I. Vernadsky University, 4 Vernadsky Ave., \\ 95007 Simferopol, Crimea, Ukraine' e-mail: alexeyev@ccssu.crimea.ua
}

Received: 08.11.2007

\begin{abstract}
.
We study the structure of $l=1$ modes in strongly elliptical coiled weakly guiding optical fibres. We establish analytically the expressions for the modes and their polarization corrections. We show that, at certain parameters of the fibre helix, the $l=1$ modes are represented in the local Frenet frame by uniform elliptically polarized fields. We demonstrate that the modes turn into circularly polarized fields if the coiling-induced perturbation becomes larger than the intrinsic spinorbit coupling. In this case the propagation constants comprise geometrically induced terms proportional to the spin angular momentum of the mode and a topological phase appears in the system. We show that the presence of such a geometric phase exhibits itself in the rotation of polarization plane of LP mode excited in the fibre. The rotation angle is found to be equal to the solid angle subtended by the coil.
\end{abstract}

Keywords: helical fibre, Berry's phase, topological phase, elliptical fibre.

PACS: 42.25.Bs, 42.81.Q

UDC: 535.1

\section{Introduction}

Coiled optical fibres hold a special place in modern optics in connection with demonstrations of topological phase effects in the optical systems. Historically, the first and the most easy-to-grasp (though not easy-to-interpret) example of the presence of Berry's topological phase [1, 2] has been provided be Tomita and Chiao [3]. In their classical experiment they have demonstrated that a linearly polarized light, being transported along a coiled single-mode fibre, changes the direction of its polarization in the local frame of reference connected with the fibre. The angle between polarizations of the input and output beams has been found to be equal to the solid angle subtended by one coil of the fibre. Simultaneously, it has been shown that the effect is associated with the evolution of photon momentum in the momentum space [4]. On a classical level, this effect has much in common with the well-known rotation of oscillation plane of the pendulum transported along a closed trajectory over a sphere [5], the polarization plane playing a role of the oscillation plane in this analogy. Although the first explanation of the mentioned effect 
has involved quantum-mechanical language [5], it has been argued that its nature has a purely classical geometric origin $[6,7]$.

Later on, such the topological rotation has been demonstrated for the images transported along curved spatial trajectories [8, 9]. An analogous topological effect has been discovered by Kataevskaya et al. in coiled fibres [10]. In this case the geometric phase manifests itself through a rotation of speckle patterns. However, until quite recently, it has not been realized that those image rotations are closely linked with a special kind of Berry's phase related to the beam with non-zero intrinsic orbital angular momentum (OAM), namely, optical vortices (OVs) [11, 12]. Generally speaking, OAM-connected Berry's phase is inherent in any fields that possess the OAM. To the best of our knowledge, the presence of such a type of topological phase has been demonstrated for quantum scalar-field vortices transported along trajectories with non-zero torsion [13]. For vectorial fields, such the spatial evolution of vortices is also accompanied by appearance of another type of topological phase linked to spin angular momentum (SAM) inherent in vectorial fields. This type of Berry's phase takes place for circularly polarized OVs (abbreviated as CVs) $|\sigma, l\rangle$, where $\sigma= \pm 1$ denotes the polarization state and $l$ stands for the orbital number of the vortex (its topological charge [14]). As has been first shown by Bliokh [15], upon evolution along a curved trajectory in the empty space, the OV $|\sigma, l\rangle$ acquires an additional phase, whose value $\gamma_{B}$ is determined by

$$
\gamma_{B}=-(\sigma+l) \Omega,
$$

where $\Omega$ is the solid angle subtended by the wave-vector trajectory in the momentum space. This expression naturally generalizes the Berry's result $\left(\gamma_{B}=-\sigma \Omega\right)$ to the case of photons with OAM. It should be specially noted that we have $\sigma \neq 0$ in Eq. (1), which can be attributed to non-zero value of the SAM of photons. Later this expression has been confirmed for the OVs propagating in the coiled few-mode optical fibres [16]. Quite recently, it has been demonstrated that one can implement the situation of scalar-field OVs in anisotropic coiled fibres, where the spin component of the total angular momentum (AM) is in effect suppressed by material anisotropy [17]. In this case the Berry's phase $\gamma_{B}=-l \Omega$ arises solely due to intrinsic OAM of the vortex beam.

Along with changes in the topological phase, the physical manifestation of topological effects connected with this type of geometric phase changes, too. For the OAMbearing beams this phase manifests itself not only through polarization rotation [16], but also as a rotation of intensity patterns at the output fibre end $[16,17]$. Exactly this type of topological effects has been reported in [8-10], which is also in compliance with theoretical predictions made in [15]. It should be emphasized that the presence of these effects depends on the AM of partial OVs, which the beam consists of, rather than on the total AM.

Ukr. J. Phys. Opt. 2008, V9, №1 
The researches have revealed that the presence of topological phase in coiled fibres is closely related to the mode structure of those fibres. For example, in the ideal coiled fibres, where the modes are represented by CVs, the geometric phase obeys the Bliokh's relation given by Eq. (1) $[16,18]$. In anisotropic coiled single-mode fibres the topological phase can be essentially suppressed by the anisotropy $[6,19]$. The material anisotropy can also suppress the topological Berry phase connected with higher-order modes in fewmode coiled fibres [17]. It is remarkable that the topological effects appear just at those values of anisotropy (i.e., perturbation), for which the fibre modes assume the structure of AM-bearing fields. To prove this indisputable connection between the AM and the topological phase, studying of other types of perturbed coiled fibres would be of interest.

One of the most widely studied types of perturbed fibres is an elliptical fibre [20]. As established in previous works, an elliptical deformation of cross-section of a coiled single-mode fibre has the same effect on the mode structure as local anisotropy does [21]. Namely, it causes hybridization of dynamical phase (i.e., the one which would have been present in a straight elliptical fibre) and a pure geometric phase (see also [19]). This leads to "blurring" of topological effects in the perturbed coiled single-mode fibres. A fundamental nature of this effect has been exhaustively explained by Chiao and Wu [4]. According to their explanation, adiabaticity condition should hold for the propagation of photons in order to ensure implementation of the geometric phase. This means that the chirality of a given photon has to remain constant upon its propagation. For the straight single-mode elliptical fibres, as well as for the anisotropic ones, polarization of the beam changes along the fibre, and the photon chirality is not conserved [20]. Naturally, this should manifest itself in the perturbed coiled fibres through diminishing of the topological effects.

The situation proves to be quite different for higher-order modes of the perturbed coiled fibres. Quite counter-intuitively, for the regimes of large anisotropy the topological phase correction hybridizes with the terms induced by spin-orbit interaction (SOI), whose absolute values are much less in the order of magnitude than those of the anisotropyinduced terms [17]. That is why the topological effects are easily observable in highly anisotropic fibres. In this respect it would be interesting to study the case of perturbed coiled fibre, whose local transverse cross-section is subjected to strong elliptical deformation.

The aim of the present paper is to study the structure of $l=1$ modes of the highly elliptical weakly guiding coiled optical fibres. We also investigate the topological effects connected with propagation of those modes along the fibre. We demonstrate that the $l=1$ modes are represented by elliptically polarized fields that bear intrinsic AM of a purely spin origin. We also prove that those modes turn into circularly polarized fields at some values of twisting parameter. In this case the propagation of modes along the fibre is accompanied by appearance of the topological phase, which resembles that present in the coiled single-mode fibres. 


\section{The model and basic equations}

Coiled fibres form a special class of optical fibres. Their main practical applications are related to the fact that geometrically induced corrections to the propagation constants of their modes are independent of external parameters such as temperature, etc. Theoretical researches have revealed that the fundamental modes of ideal coiled fibres are represented by circularly polarized fields [7, 22]. The perturbation effect on the mode structure of coiled single-mode fibres has been studied in $[6,19,21]$. Those studies have established that the $l=0$ modes of anisotropic and elliptical coiled fibres are given by locally elliptically polarized fields. As for the higher order modes, they have been studied only for the ideal coiled $[16,18]$ and anisotropic [17] fibres.

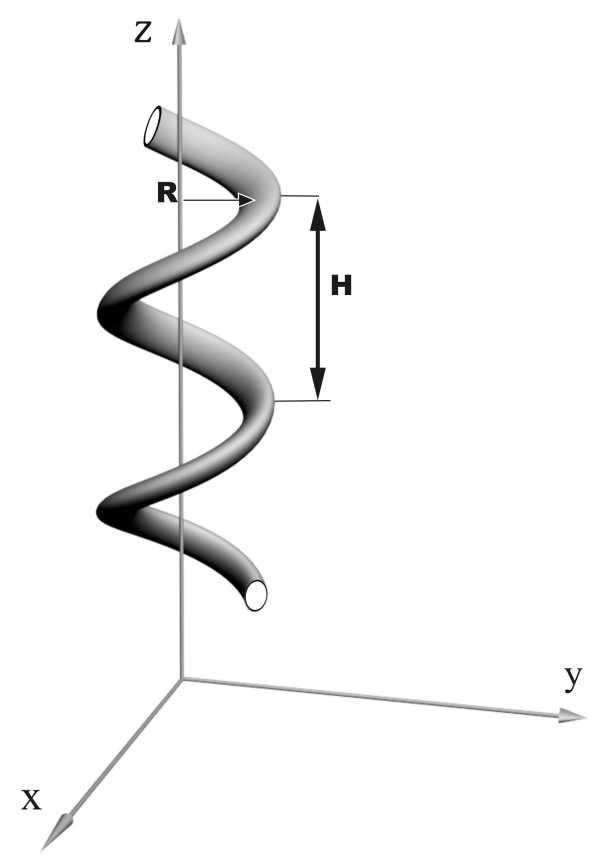

Fig. 1. The model of a helical optical fibre with elliptical cross-section; $H$ is the pitch, $R$ is the offset, $(x, y, z)$ is the lab frame.
Let us consider a helically wound fibre whose transverse local cross-section has an elliptic form (see Fig. 1). The geometry of coiling is described by the offset $\mathrm{R}$ and the pitch $\mathrm{H}$. The parameters of the deformation ellipse will be specified in what follows. Of course, any mode of a bent fibre represents rather a metastable state due to the presence of inevitable radiation losses [23]. However, in our model we will disregard such the losses, as well as the effects of mechanical stresses, which may cause various photoelastic phenomena (see also [17]). As is known, a monochromatic light in optical fibre is described by the so-called vector wave equation [20],

$$
\left(\vec{\nabla}^{2}+n^{2}(x, y, z) k^{2}\right) \mathbf{E}(x, y, z)=-\vec{\nabla}\left(\mathbf{E}(x, y, z) \quad \vec{\nabla} \ln n^{2}(x, y, z)\right),
$$

where $\nabla=(\partial / \partial x, \partial / \partial y, \partial / \partial z), k=2 \pi / \lambda, \lambda$ is the light wavelength and $\mathbf{E}$ the electric field. The refractive index is conventionally written in the form

$$
n^{2}(x, y, z)=n_{c o}^{2}(1-2 \Delta f(x, y, z)),
$$

where $n_{c o}$ and $n_{c l}$ are the refractive indices respectively for the core and cladding and the height $\Delta$ of the refractive index profile is

$$
\Delta=\frac{n_{c o}^{2}-n_{c l}^{2}}{2 n_{c o}^{2}} .
$$


The profile function $f(x, y, z)$ determines the distribution of the refractive index. In this work we study weakly guiding fibres, for which $\Delta<<1$.The term in the r. h. s. of Eq. (2) is usually called as a gradient one. Propagation of light in the coiled fibres may be studied by various methods, including the Mueller matrix formalism [24] and the Jones matrix technique [25]. One of them is solving the wave equation (2) using the local coordinates. It would be natural to use the coordinates $(r, \varphi, s)$ shown in Fig. 2 [22], where $r$ and $\varphi$ are the polar coordinates in the fibre cross-section and $S$ the length of central line of the fibre.

Analytically, elliptical deformation of the fibre cross-section can be introduced by a number of methods. The most widespread technique for the straight elliptical fibres is passing to the elliptic coordinates. It involves complications on characteristic equation for the propagation constant and is preferable for the fibres with large $\Delta$ values. In the case of weakly guiding fibres, the ellipticity is much easily introduced via a simple coordinate transformation in $n(\vec{r})$. According to this method, one should make the following change of variables in the function

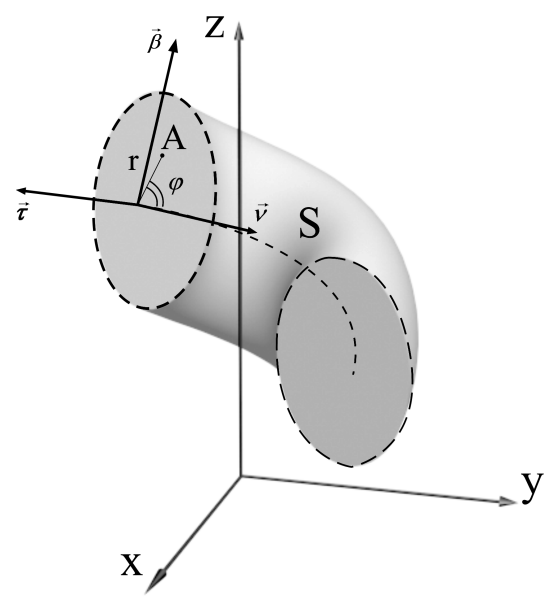

Fig. 2. Local helical coordinates $(r, \varphi, s)$ in the Frenet frame $\mathbf{v}, \boldsymbol{\beta}, \boldsymbol{\tau}$, where $\boldsymbol{v}$ is the unit vector of the principal normal, $\boldsymbol{\beta}$ is the unit vector of the binormal, $\tau$ is the unit vector in the tangent direction. $\tilde{n}(x, y) \equiv f\left(\sqrt{x^{2}+y^{2}}\right)$ that describes the refractive index of ideal fibres [26]:

$$
\begin{aligned}
& x \rightarrow x(1+\delta), \\
& y \rightarrow y(1-\delta) .
\end{aligned}
$$

Here $\delta<<1$ is supposed to be the deformation parameter. It is associated with the eccentricity $e$ of the ellipse, on which the value of $n$ is constant, through a simple relation

$$
e=\frac{2 \sqrt{\delta}}{1+\delta}
$$

Under the transformation given by Eq. (5) the modified refractive index $\tilde{n}$ of ideal fibre acquires dependence on the azimuth $\varphi$ and reads in the polar coordinates $(r, \varphi)$ as

$$
n^{2} \approx \tilde{n}^{2}-2 n_{c o}^{2} \Delta \delta \cdot r \frac{\partial \tilde{f}}{\partial r} \cos 2 \varphi,
$$

where the tilde denotes an axially symmetric function. Note that for simplicity we use here the same notation for the polar coordinates as for the helical ones. As a matter of fact, introducing the ellipticity of a coiled fibre should imply using Eq. (7) for the refrac- 
tive index distribution, with the only exception that the variables in Eq. (7) should be treated as those of the helical coordinates. Now we are in a position to obtain the vector wave equation for the helical elliptical fibre.

To recast Eq. (2) in the helical coordinates $(r, \varphi, s)$, one has to use their link with the Cartesian coordinates $(x, y, z)[27]$ :

$$
\begin{aligned}
& x=R \cos K \mathrm{~s}-r \cos \varphi \cos K \mathrm{~s}+r v \sin \varphi \sin K \mathrm{~s}, \\
& y=R \sin K \mathrm{~s}-r \cos \varphi \sin K \mathrm{~s}-r v \sin \varphi \cos K \mathrm{~s}, \\
& z=v s+r R K \sin \varphi,
\end{aligned}
$$

where $K=2 \pi /\left(H^{2}+(2 \pi R)^{2}\right)^{1 / 2}$ and $v=H K / 2 \pi$. Basing on the standard relation $\vec{\nabla}^{2} \mathbf{A}=\operatorname{grad} \operatorname{div} \mathbf{A}-\operatorname{rot} \operatorname{rot} \mathbf{A}$, one can recast the term $\vec{\nabla}^{2} \mathbf{E}$ in Eq. (2) using the wellknown formulae of differential geometry [28]:

$$
\begin{gathered}
\operatorname{div} \mathbf{A}=\frac{1}{\sqrt{\mathrm{G}}} \frac{\partial}{\partial \mathrm{x}^{i}}\left(\mathrm{~A}_{i} \sqrt{\frac{G}{g_{i i}}}\right), \\
(\nabla \Phi)_{k}=\sqrt{g_{k k}} g^{k i} \frac{\partial \Phi}{\partial x^{i}}, \\
(\operatorname{rot} \mathbf{A})_{i}=\sqrt{\frac{g_{i i}}{G}}\left[\frac{\partial}{\partial x_{j}}\left(g_{k n} \frac{A_{n}}{\sqrt{g_{n n}}}\right)-\frac{\partial}{\partial x_{k}}\left(g_{j n} \frac{A_{n}}{\sqrt{g_{n n}}}\right)\right],
\end{gathered}
$$

where the metric tensor $g_{i j}$ for the helical coordinates reads as

$$
g_{i j}=\left(\begin{array}{ccc}
1 & 0 & 0 \\
0 & r^{2} & r^{2} v \\
0 & r^{2} v & g_{33}
\end{array}\right)
$$

Here we have used the notations $g_{33}=(1-r \kappa \cos \varphi)^{2}+r^{2} v^{2}$ and $G=\operatorname{det} g_{i k}$, with $\kappa=\frac{4 \pi^{2} R}{H^{2}+(2 \pi R)^{2}}$ being the curvature of central line of the fibre and $v=\frac{2 \pi H}{H^{2}+(2 \pi R)^{2}}$ its torsion. In the expression for curl one has $(i, j, k)=(1,2,3)$ and all their cyclic permutations.

A standard simplification made in the case of weakly coiled $\left(r_{0} \kappa \ll 1, r_{0}\right.$ being the core radius) and weakly guiding $(\Delta<<1)$ fibres is disregarding the longitudinal component $E_{S}$ (directed along $\boldsymbol{\tau}$ ), when compare with the transverse one $E_{t}: E_{t}>E_{S}$. This enables disentangling the obtained rigorous equations into separate equations for $E_{t}$ and $E_{s}$. The resulting system in $\mathbf{E}_{t}$ proves to be translation invariant in $s$, thus allowing one to search for its solution in a factorized form: 


$$
\mathbf{E}_{t}(r, \varphi, s)=\mathbf{e}_{t}(r, \varphi) \exp (i \beta s)
$$

where $\beta$ is a propagation constant. After cumbersome calculations one can obtain a matrix equation in the ket:

$$
|\Phi\rangle_{h} \equiv\left(\begin{array}{c}
e_{r}(r, \varphi) \\
e_{\varphi}(r, \varphi)
\end{array}\right)
$$

It consists of the component of $\mathbf{e}_{t}$ on the local basis $\mathbf{n}_{r}, \mathbf{n}_{\varphi}$, which is indicated by the subscript $h$.

The desired equation looks as

$$
\left(\hat{H}_{0}+\hat{V}\right)|\Phi\rangle_{h}=\beta^{2}|\Phi\rangle_{h}
$$

where

$$
\hat{H}_{0}=\left(\frac{\partial^{2}}{\partial r^{2}}+\frac{1}{r} \frac{\partial}{\partial r}+\frac{1}{r^{2}} \frac{\partial^{2}}{\partial \varphi^{2}}-\frac{1}{r^{2}}+k^{2} \tilde{n}^{2}(r)\right) \hat{\sigma}_{0}+\frac{2 i}{r^{2}} \frac{\partial}{\partial \varphi} \hat{\sigma}_{2},
$$

with $\sigma_{i}$ being the Pauli matrix. It is worth noticing that $\hat{H}_{0}$ describes the propagation of light in an ideal straight fibre in the case if local basis of cylindrical-polar coordinates is chosen. The perturbation operator $\hat{V}$ comprises the geometric effects, together with the effects of ellipticity. It has the form

$$
\begin{aligned}
& \hat{V}=\left\{\left(-\kappa \cos \varphi-r \kappa^{2} \cos ^{2} \varphi\right) \frac{\partial}{\partial r}-\left(2 r \kappa \cos \varphi+3 r^{2} \kappa^{2} \cos ^{2} \varphi\right) \beta^{2}\right\} \hat{\sigma}_{0} \\
& +\left\{-2 k^{2} n_{c o}^{2} \delta \Delta \tilde{f}_{r}^{\prime} \cos 2 \varphi+v^{2} \frac{\partial^{2}}{\partial \varphi^{2}}+\frac{1}{\sqrt{G}}\left[\kappa \sin \varphi \frac{\partial}{\partial \varphi}-2 i \beta\left(r v+r^{2} \kappa v \cos \varphi\right) \frac{\partial}{\partial \varphi}\right]-\frac{\kappa^{2}}{2}\right\} \hat{\sigma}_{0} \\
& +\frac{r \kappa}{\sqrt{G}}\left[\frac{1}{2} \kappa \sin 2 \varphi+\beta r v \cos \varphi\right] \hat{\sigma}_{1}+\frac{\kappa}{\sqrt{G}}\left[-i \sin \varphi-i \beta r^{2} v \cos \varphi\right] \hat{\sigma}_{2} \\
& +\left[-\kappa^{2} \cos ^{2} \varphi+\frac{r^{2}}{\sqrt{G}} \kappa v i \beta \sin \varphi+\frac{v^{2} K^{2}}{2}\right] \hat{\sigma}_{3} \\
& +\frac{1}{2}\left\{\frac{\partial}{\partial r} \psi_{r}^{\prime} \hat{\sigma}_{0}+\frac{\psi_{r}^{\prime}}{r} \frac{\partial}{\partial \varphi} \hat{\sigma}_{1}-\frac{i \psi_{r}^{\prime}}{r} \frac{\partial}{\partial \varphi} \hat{\sigma}_{1}+\frac{\partial}{\partial r} \psi_{r}^{\prime} \hat{\sigma}_{3}\right\}
\end{aligned}
$$

Here $\psi=\ln \tilde{n}^{2}$ and the prime stands for the derivative with respect to $r$. The terms in Eq. (17) placed in the last curly brackets originate from the gradient term on the r. h. s. of Eq. (2) taken in the straight fibre approximation. In this limit the helical coordinates coincide with the cylindrical-polar ones. The gradient term may be written as

$$
\vec{\nabla}_{t}\left(\mathbf{E}_{t} \cdot \vec{\nabla}_{t} \ln n^{2}\right)=\mathbf{n}_{r} \vec{\nabla}_{r}\left(\psi_{r}^{\prime} E_{r}\right)+\mathbf{n}_{\varphi} \frac{\psi_{r}^{\prime}}{r} \vec{\nabla}_{\varphi} E_{r},
$$


where $\mathbf{n}_{r}$ and $\mathbf{n}_{\varphi}$ are the unit vectors of cylindrical-polar coordinates. In a matrix form Eq. (18) stands for $\left(\begin{array}{cc}\nabla_{r} \psi_{r}^{\prime} & 0 \\ \frac{\psi_{r}^{\prime}}{r} \nabla_{\varphi} & 0\end{array}\right) \cdot\left(\begin{array}{c}E_{r} \\ E_{\varphi}\end{array}\right)$, which can be decomposed in the Pauli matrix just as the group of terms mentioned above. These terms essentially describe SOI in the optical fibres $[29,30]$, i.e. a cross influence of spin (polarization) properties of an electromagnetic wave and orbital (spatial) characteristics of its energy motion upon propagating. In terms of geometrical optics, the SOI manifests itself as a mutual influence of the polarization and the trajectory of ray motion. The SOI lies in the basis of a number of optical phenomena, such as optical Magnus effect, etc. [31, 32]. Mathematically the SOI is implemented through the operators that act simultaneously on both the angular and spin variables. In fibres the SOI results in difference in the propagation constants of OVs with the same orbital number (at $l \geq 2$ ) and the opposite signs of their circular polarizations. Mark that by using the gradient term in the straight fibre approximation, we have neglected the influence of geometry on the SOI. The third term in $\hat{V}$ describes the effect of the ellipticity, whereas the other terms have a purely geometric origin.

\section{The structure of $l=1$ modes}

Mathematically, Eq. (15) has a form of eigenvalue equation. As is well established, utilization of the perturbation theory proves to be fruitful for the weakly guiding fibres [33]. In our case the operator $\hat{V}$ should be taken as a perturbation. Then the zeroapproximation equation should be written as $\hat{H}_{0}|\Phi\rangle_{h}=\tilde{\beta}_{l}^{2}|\Phi\rangle_{h}$, where $\not{\beta}$ is the scalar propagation constant. Further we will show that this constant coincides with the standard scalar propagation constant of ideal straight fibres. Since the operator $\hat{H}_{0}$ is translation invariant in $\varphi$, it is possible to search for its solution in a factorized form:

$$
|\Phi\rangle_{h}=\Phi(r) \exp (i m \varphi)\left|\Phi_{0}\right\rangle_{h}
$$

where $m=0, \pm 1, \pm 2 \ldots$ and $\left|\Phi_{0}\right\rangle_{h}$ is some constant vector. It is convenient to chose the eigenvectors of the matrix $i \hat{\sigma}_{2}$ for $\left|\Phi_{0}\right\rangle_{h}:\left|\Phi_{+}\right\rangle_{h} \equiv\left(\begin{array}{l}1 \\ i\end{array}\right)$ belonging to the eigenvalue $i$ and $\left|\Phi_{-}\right\rangle_{h} \equiv\left(\begin{array}{l}1 \\ -i\end{array}\right)$ belonging to the eigenvalue $-i$. Then the eigenvectors of $\hat{H}_{0}$ assume the form

$$
\left|F_{ \pm}\right\rangle_{h}=F_{m}^{ \pm}(r) \exp (i m \varphi)\left|\Phi_{ \pm}\right\rangle_{h}
$$

where $F_{m}^{ \pm}$satisfies the following equation:

$$
\left(\frac{d^{2}}{d r^{2}}+\frac{1}{r} \frac{d}{d r}+k^{2} \tilde{n}^{2}-\frac{(m \mp 1)^{2}}{r^{2}}-\tilde{\beta}^{2}\right) F_{m}^{ \pm}(r)=0 .
$$


Evidently, the function $F_{m}^{ \pm}$represents a radial function $F_{l}$ of the ideal straight fibre if only $l \equiv m \mp 1$, so that $\widetilde{\beta}$ should acquire a natural subscript $l$.

It is easily proven that each eigenvalue $\tilde{\beta}_{l}^{2}$ is degenerate. Indeed, the value $l=0$ can be obtained at $m=1$ if the upper sign is chosen. If $m=-1$, one has to choose the lower sign in order to get $l=0$. So, there are two eigenvectors that belong to the eigenvalue $\tilde{\beta}_{0}$ :

$$
\left|F_{ \pm}^{0}\right\rangle_{h}=F_{0}(r) \exp ( \pm i \varphi)\left|\Phi_{ \pm}\right\rangle_{h}
$$

Let us emphasize that, in this way, the $l=0$ modes given by Eq. (22) become dependent on $\varphi$, which should be attributed to locality of the basis $\mathbf{n}_{r}, \mathbf{n}_{\varphi}$. The degree of degeneracy doubles if $l \neq 0$ : the case $|l|=1$ can be obtained in four different ways: $m=2 ; 0$ for the upper sign and $m=-2 ; 0$ for the lower one. This gives four eigenvectors that belong to the same eigenvalue $\tilde{\beta}_{1}^{2}$ :

$$
\begin{array}{ll}
|1\rangle_{h}=F_{1}(r) \exp (2 i \varphi)\left|\Phi_{+}\right\rangle_{h}, & |2\rangle_{h}=F_{1}(r)\left|\Phi_{+}\right\rangle_{h}, \\
|3\rangle_{h}=F_{1}(r) \exp (-2 i \varphi)\left|\Phi_{-}\right\rangle_{h}, & |4\rangle_{h}=F_{1}(r)\left|\Phi_{-}\right\rangle_{h} .
\end{array}
$$

In a similar manner, for arbitrary $l \neq 0$ one obtains the following eigenvectors (written in the explicit form) that belong to the eigenvalue $\tilde{\beta}_{l}^{2}$ :

$$
\begin{aligned}
|1\rangle_{h} & =\mathrm{e}^{i(l+1) \varphi}\left(\begin{array}{l}
1 \\
i
\end{array}\right) F_{l}(r), \quad|2\rangle_{h}=\mathrm{e}^{i(1-l) \varphi}\left(\begin{array}{l}
1 \\
i
\end{array}\right) F_{l}(r), \\
|3\rangle_{h} & =\mathrm{e}^{i(-1-l) \varphi}\left(\begin{array}{l}
1 \\
-i
\end{array}\right) F_{l}(r), \quad|4\rangle_{h}=\mathrm{e}^{i(l-1) \varphi}\left(\begin{array}{l}
1 \\
-i
\end{array}\right) F_{l}(r) .
\end{aligned}
$$

For the step-index fibres $F_{l}$ is the Bessel function. Though the vectors given by Eqs. (22)-(24) are represented in the helical frame of reference $\mathbf{n}_{r}, \mathbf{n}_{\varphi}$, it is also convenient to have their form in the Frenet frame $\mathbf{v}, \boldsymbol{\beta}$ :

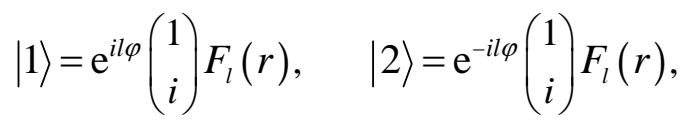

$$
\begin{aligned}
& |3\rangle=\mathrm{e}^{-i l \varphi}\left(\begin{array}{l}
1 \\
-i
\end{array}\right) F_{l}(r), \quad|4\rangle=\mathrm{e}^{i l \varphi}\left(\begin{array}{l}
1 \\
-i
\end{array}\right) F_{l}(r) .
\end{aligned}
$$

In what follows, we will represent the final results in the Frenet basis, so that $|\Phi\rangle \equiv\left(\begin{array}{l}e_{v}(r, \varphi) \\ e_{\beta}(r, \varphi)\end{array}\right)$. The solutions given by Eq. (25) should be recognized as the CVs $|\sigma, l\rangle:$

$$
|1\rangle=|1, l\rangle, \quad|2\rangle=|1,-l\rangle, \quad|3\rangle=|-1,-l\rangle, \quad|4\rangle=|-1, l\rangle .
$$


In terms of standard fibre modes, the CVs (26) may be represented as

$$
| \pm 1, \pm l\rangle=H E_{l+1, n}^{e v} \pm i H E_{l+1, n}^{o d},|\mp 1, \pm l\rangle=E H_{l-1, n}^{e v} \pm i E H_{l-1, n}^{o d}
$$

where the upper and lower signs should be taken simultaneously on the both sides of these relations. Note that for simplicity we omit throughout the text the radial indices $n$, as well as the exponentials comprising the propagation constants, thus assuming that they do not vary within the modes (25).

Since there is a four-fold degeneracy of the spectrum of $\hat{H}_{0}$ (at $\left.l \neq 0\right)$, one should use the perturbation theory with degeneracy while obtaining the polarization corrections to the propagation constant $\tilde{\beta}_{l}^{2}$ and establishing the structure of modes [33]. According to that theory, one needs to build matrix of the operator $\hat{V}$ on the basis of eigenvectors (24) that belong to the same eigenvalue $\tilde{\beta}_{l}^{2}$. The matrix elements are found as $V_{i j}=\langle\hat{V} j\rangle$, where the scalar product is defined by

$$
\langle\Phi \mid \Psi\rangle=\int_{0}^{\infty} \int_{0}^{2 \pi}\left(\begin{array}{ll}
\Phi_{r}^{*} & \Phi_{\varphi}^{*}
\end{array}\right)\left(\begin{array}{c}
\psi_{r} \\
\psi_{\varphi}
\end{array}\right) r d r d \varphi .
$$

The structure of the modes is readily obtained from the standard secular equation

$$
V \mathbf{x}=\Delta \beta^{2} \mathbf{x},
$$

where $\Delta \beta^{2}=\beta^{2}-\tilde{\beta}_{l}^{2}$.

For the case of $l=1$ modes, the perturbation matrix $V_{1}$ is given by

$$
V_{1}=\left(\begin{array}{cccc}
A_{1}+4 \beta v-4 v^{2} & D_{1} & 0 & 0 \\
D_{1} & B_{1} & 0 & B_{1} \\
0 & 0 & A_{1}-4 \beta v-4 v^{2} & D_{1} \\
0 & B_{1} & D_{1} & B_{1}
\end{array}\right),
$$

where for the step-index fibres we have

$$
\begin{aligned}
& A_{1}=\frac{\Delta}{Q_{1} r_{0}^{2}}\left(F_{1} F_{1}^{\prime}-F_{1}^{2}\right)_{R=1} \\
& B_{1}=\frac{\Delta}{Q_{1} r_{0}^{2}}\left(F_{1}^{2}+F_{1} F_{1}^{\prime}\right)_{R=1}, \\
& D_{1}=-k^{2} n_{c o}^{2} \delta \Delta F_{1}^{2}(R=1) / Q_{1} .
\end{aligned}
$$

Here the normalization coefficient reads as $Q_{1}=\int_{0}^{\infty} R F_{1}^{2}(R) d R$, with $R=r / r_{0}$. The matrix (30) describes the combined action of different physical factors: the constants $A_{1}, B_{1}$ represent in effect the SOI, the terms with the torsion account for the principal 
contribution of geometric effects and, finally, $D_{1}$ describes the ellipticity. It is helpful to notice that the matrix $V_{1}$ has the same structure as the matrix (15) from the work [34], which describes the modes of twisted elliptical fibres. From this point of view, the torsion $v$ is analogous to the twist parameter q. Physically, this means that both the torsion and twist give rise to the appearance of circular birefringence in the system.

Unfortunately, it proves impossible to obtain a compact analytical expression for eigenvectors and eigenvalues of the matrix $V_{1}$ at arbitrary values of its parameters. However, in the most practically relevant case of relatively large ellipticity, which is implemented at $\left|D_{1}\right| \gg 4 \beta v,\left|A_{1}\right|,\left|B_{1}\right|$, the modes are easily found to be

$$
\begin{aligned}
& \left|\psi_{1}\right\rangle=F_{1}(r)\left(\begin{array}{c}
\sin \theta \\
i \cos \theta
\end{array}\right) \cos \varphi, \quad\left|\psi_{2}\right\rangle=F_{1}(r)\left(\begin{array}{c}
\cos \theta \\
-i \sin \theta
\end{array}\right) \cos \varphi, \\
& \left|\psi_{3}\right\rangle=F_{1}(r)\left(\begin{array}{c}
\cos \theta \\
i \sin \theta
\end{array}\right) \sin \varphi, \quad\left|\psi_{4}\right\rangle=F_{1}(r)\left(\begin{array}{c}
\sin \theta \\
-i \cos \theta
\end{array}\right) \sin \varphi,
\end{aligned}
$$

where $\cot 2 \theta=-\left|B_{1}\right| / 4 q \tilde{\beta}$ and $\pi / 4 \leq \theta \leq \pi / 2$.

The corresponding propagation constants of these modes may be written as

$$
\begin{aligned}
& \beta_{1,2}=\tilde{\beta}+\frac{1}{4 \tilde{\beta}}\left(2\left|D_{1}\right|+A_{1}+B_{1} \pm \sqrt{16 v^{2} \tilde{\beta}^{2}+B_{1}^{2}}\right) \\
& \beta_{3,4}=\tilde{\beta}+\frac{1}{4 \tilde{\beta}}\left(-2\left|D_{1}\right|+A_{1}+B_{1} \pm \sqrt{16 v^{2} \tilde{\beta}^{2}+B_{1}^{2}}\right)
\end{aligned}
$$

Note that the modes (32) are written in the Frenet frame. Besides, we have used the well-known relationship [20]

$$
\delta \beta=\beta-\tilde{\beta} \approx \Delta \beta^{2} / 2 \tilde{\beta},
$$

while obtaining Eq. (33). The dependence of polarization corrections on the offset is given in Fig. 3.

As follows from Eq. (32), the modes are elliptically polarized fields. The mode structure is governed by the ratio of the vectors components $\sin \theta$ and $\cos \theta$. Obviously, these modes are represented by circularly polarized fields at $\sin \theta \approx \pm \cos \theta$. This occurs if the SOI constant $B_{1}$ is much less than the coiling-induced term in the matrix $V_{1}$. In the opposite case the modes are given by the well-known LP modes of ideal fibres [20], except for that these modes trace adiabatically orientation of the Frenet frame. This situation is much the same as that taking place in the twisted elliptical fibres [35]. The modes given by Eq. (32) may be represented as a sum of left and right circularly polarized fields, e.g.

$$
\left|\psi_{1}\right\rangle=\frac{F_{1}(R)}{2}\left\{(\cos \theta+\sin \theta)\left|\Phi_{+}\right\rangle+(\cos \theta-\sin \theta)\left|\Phi_{+}\right\rangle\right\} \cos \varphi
$$

It follows from this relation that the relative power $N_{ \pm} \propto\left|E_{ \pm}\right|^{2}$ ( $E_{ \pm}$being the partial 
amplitude stored in the corresponding circularly polarized field) is proportional to $(\cos \theta \pm \sin \theta)^{2}$. The ratio of the powers stored in the right and left circularly polarized constituents of the first mode (32),

$$
\frac{N_{+}}{N_{-}}=\tan ^{2}(\theta-\pi / 4)
$$

is displayed in Fig. 4.

\section{Topological phase in the coiled elliptical fibres}

Propagation of light along the coiled fibres is sometimes connected with the effects that do not depend upon dynamical factors associated with the nature of fibre material, the structure of the fibre and the like. Some effects depend only on geometric configuration of the fibre and are highly insensitive to variations of intrinsic fibre parameters. Among those effects one should mainly list the polarization rotation and the topological image
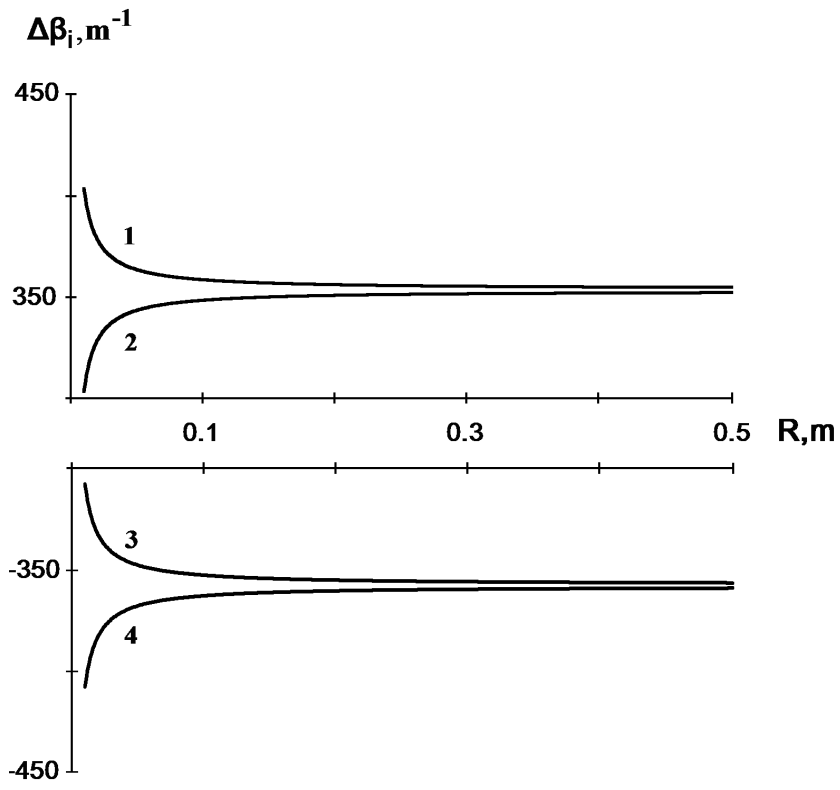

$\left|\mathbf{E}_{+}\right|^{2} /\left|\mathbf{E}_{-}\right|^{2}$

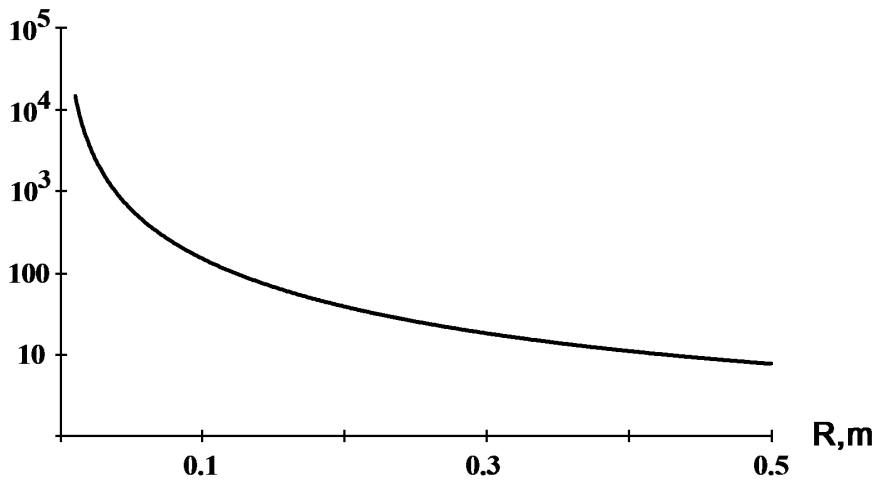

Fig. 3. corrections $\Delta \beta_{i}=\beta_{i}-\tilde{\beta}$ versus offset for modes (32) of a coiled elliptical fibre, waveguide parameter $\quad V=4.2$, $\delta=0.05, . \quad$ The pitch $H=2 \pi R$ is taken to provide maximal torsion at a given offset [18].
Fig. 4. The ratio of the powers $\left|E_{ \pm}\right|^{2}$ stored in right and left circularly polarized constituents of the first mode (32) versus offset (in semi-logarithmic scale); waveguide parameter $\quad V=4.2$, $\delta=0.05, H=2 \pi R$. 
rotation. Historically, the former kind of topological effects has become a first example of geometric phase in optics (somewhat later, the Pancharatnam phase [36] has been recognized to be a manifestation of the Berry's phase [37]). As for the latter, though the topological rotation of images has also been reported in a number of experiments [8-10], until quite recently it has mainly been treated in the framework of spin-connected Berry's phase. Meanwhile, it has been demonstrated [13, 15-17] that the image rotation is connected with a special kind of Berry's phase inherent in photons with intrinsic OAM. In the works quoted above the principles of observation of effects associated with such an "orbital" topological phase have been outlined. It has been established that the presence of constituent fields with the OAM (and the OVs) leads to rotation of interference patterns. In the case of strongly anisotropic coiled fibres this image rotation is not accompanied by the polarization rotation [17] and is the only topological effect. In this section we provide argumentation that the situation for strongly elliptical coiled fibres is reverse: the only geometric effect consists here in the polarization rotation.

To prove this statement let us consider excitation of a coiled elliptical fibre by a Hermite-Gaussian mode $H G_{01}$. As is known, this causes excitation of the LP fibre mode [20]. Let this LP mode has the form $F_{1}(r)\left(\begin{array}{c}\cos \varphi \\ 0\end{array}\right)$ at the input end. Then it is decomposed in the fibre into the coiled-fibre modes (32), i.e.

$$
|\Phi(s)\rangle=\sin \theta\left|\psi_{1}(s)\right\rangle+\cos \theta\left|\psi_{2}(s)\right\rangle .
$$

Note that here we include the phase exponentials into the mode structure. After the coil $\left(s_{0}=2 \pi / K\right)$ the input field $\left.\Phi(0)\right\rangle$ acquires the form $\left|\Phi\left(s_{0}\right)\right\rangle$ :

$$
\left|\Phi\left(s_{0}\right)\right\rangle=F_{1}(r) \cos \varphi\left(\begin{array}{c}
\cos \gamma-i \sin \gamma \cos 2 \theta \\
-\sin 2 \theta \sin \gamma
\end{array}\right),
$$

where $\gamma=s_{0} \sqrt{v^{2}+\left(B_{1} / 4 \tilde{\beta}\right)^{2}}$. This elliptically polarized field is characterized by the following Stokes parameters:

$$
\begin{aligned}
& S_{1}=\cos ^{2} \gamma+\sin ^{2} \gamma \cos 4 \theta, \\
& S_{2}=-\sin 2 \gamma \sin 2 \theta, \\
& S_{3}=-\sin ^{2} \gamma \sin 4 \theta .
\end{aligned}
$$

The inclination $\psi$ of the major axis of polarization ellipse is given by

$$
\tan 2 \psi=\frac{S_{2}}{S_{1}}=\frac{-\sin 2 \gamma \sin 2 \theta}{\cos ^{2} \gamma+\sin ^{2} \gamma \cos 4 \theta} .
$$

Since the r. h. s. of Eq. (33) entangles the dynamical $\left(B_{1}\right)$ and geometric $(v)$ properties of the fibre, no purely geometric effect is observable for an arbitrary relation between 
$B_{1}$ and $v$. However, the effect of coiling becomes prevailing if $4 \tilde{\beta} v>>B_{1}$ and an explicitly topological effect appears in the system. Simultaneously, the modes become circularly polarized fields. However, since this topological effect is connected with the phase of the modes rather than their structure, it would be more reliable to judge implementation of the topological effect by the corresponding phase characteristics. In Fig. 5 we have provided the ratio $4 \tilde{\beta} v>>B_{1}$ versus the offset that governs also the phase dependence of the modes given by Eq. (32). The topological effect appears at the area of the offset values where $4 \tilde{\beta} v>>B_{1}$.

Indeed, in these areas one simultaneously has $\theta \rightarrow \pi / 4$. Furthermore, from Eq. (40) it follows that $\tan 2 \psi \approx-\tan 2 \gamma \approx-\tan 2 v s_{0}$. In the same manner, as it has been done in [16], one can show that the last relation yields in that, in the laboratory frame of reference (connected with the input end of the fibre), the major axis makes the angle

$$
\psi=2 \pi(1-v)
$$

with the direction of the input beam polarization. Since $v=\cos \alpha$, where $\alpha$ is the angle between the wave vector $\mathbf{k}$ and the $\mathrm{z}$ axis, this result can also be expressed in terms of the solid angle $\Omega$ subtended by $\mathbf{k}$ in the momentum space:

$$
\psi=\Omega \text {. }
$$

One should notice that the output beam becomes linearly polarized at $4 \tilde{\beta} v>>B_{1}$, and the topological effect reduces to geometric rotation of the polarization plane, as in the case of single-mode fibres. Let us also emphasize that by exciting the mode $\propto\left(\begin{array}{c}\cos \varphi \\ 0\end{array}\right)$ at

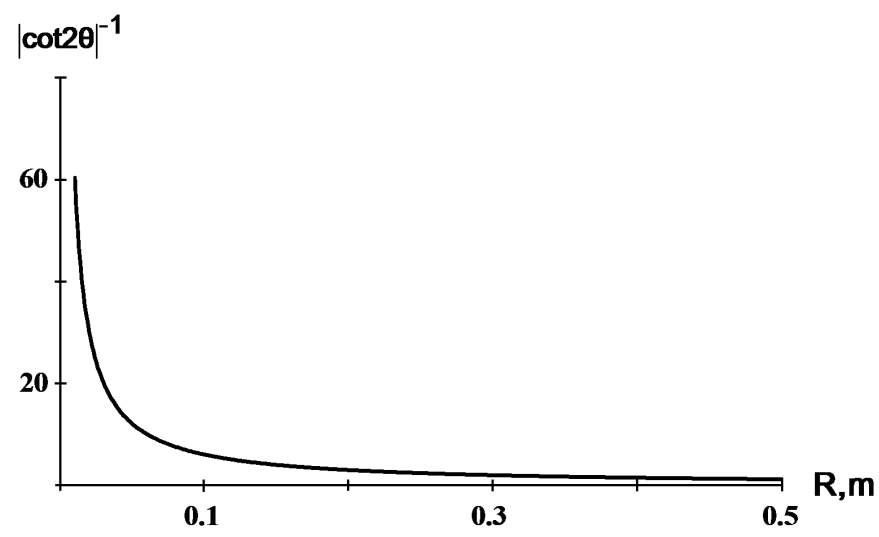

Fig. 5. Ratio $4 \tilde{\beta} v / B_{1}$ of the geometry-induced phase corrections to the SOIinduced ones versus the offset: $V=4.2, \delta=0.05, . H=2 \pi R$. The topological phase is implemented in the area where $4 \tilde{\beta} v>>B_{1}$.

the input end, we simultaneously provide that the ellipticity-induced dynamical phases, 
which are included into the structure of modes $\left|\psi_{1,2}(s)\right\rangle$ in the decomposition (37), coincide. Otherwise, no topological effect would be observed against a background of purely dynamical ones caused by the ellipticity of the fibre. For example, this will be the case for the linearly polarized $\mathrm{OV}\left(\begin{array}{c}\exp (i \varphi) \\ 0\end{array}\right)$ : the decomposition in the fibre modes (32) analogous to Eq. (37) would involve the modes $\left(\begin{array}{c}\cos \varphi \\ 0\end{array}\right)$ and $\left(\begin{array}{c}\sin \varphi \\ 0\end{array}\right)$ of different oddity, which have also different dynamical terms $\pm D_{1} / 2 \tilde{\beta}$ in their polarization correction (33).

It is not accidental that, though the $l=1$ modes of the coiled elliptical fibres involve the partial fields $\pm 1, \pm 1\rangle$ with a non-zero OAM, the topological phase does not manifest itself in the image rotation, as has been with the coiled anisotropic fibres [17]. The reason for this asymmetry can be found in the nature of AM for the coiled fibre modes in case of a strong coiling. Indeed, whereas the modes of strongly coiled anisotropic fibres carry the OAM, the corresponding modes of the coiled elliptical fibres carry the AM of spin nature. This circumstance is responsible for the fact that the coiled elliptical fibres exhibit the same topological effects as the single-mode fibres, in which photons possess only the SAM.

\section{Conclusion}

We have shown that the $l=1$ modes of strongly elliptical coiled fibres are given by the fields that maintain elliptical polarization in the local Frenet frame. If the coiling-induced perturbation becomes larger than the intrinsic SOI, the modes turn into circularly polarized fields. In this limiting case the intrinsic SAM of $l=1$ modes tends to unity and a topological effect appears in the system. We have established that such a geometric phase manifests itself through a rotation of polarization plane of the LP mode excited in the fibre. Similar to single-mode fibres, the rotation angle is found to be equal to the solid angle subtended by the fibre coil. Its value coincides with the Berry's topological phase acquired by the mode upon propagation by one fibre coil.

\section{References}

1. Berry MV, 1984. Quantum phase factor accompanying adiabatic changes. Proc Roy. Soc. Lond. A 392: 45-57.

2. Shapere A. and Wilczek F. Geometric phases in physics. World Scientific: Singapore, (1989).

3. Tomita A and Chiao RY, 1986. Observation of Berry's topological phase by use of an optical fibre. Phys. Rev. Lett. 57: 937-940.

4. Chiao RY and Wu Y-S, 1986. Manifestation of Berry's topological phase for the phton, Phys. Rev. Lett. 57: 933-936.

5. Berry MV, 1988. The geometric phase. Scient. Amer. 259: 26-34. 
6. Ross JN, 1984. The rotation of the polarization in low birefringence single-mode optical fibres due to geometric effects. Opt. Quant. Electron. 16: 455-461.

7. Berry MV, 1987. Interpreting the anholonomy of coiled light. Nature 326: 277-278.

8. Galvez EJ and Holmes CD, 1999. Geometric phase of optical rotators. J. Opt. Soc. Am. A. 16: 1981-1985..

9. Segev M, Solomon R and Yariv A, 1992. Manifestation of Berry's phase in imagebearing optical beams. Phys. Rev. Lett. 69: 590-593 .

10. Kataevskaya IV and Kundikova ND, 1995. Influence of the helical shape of a fibre waveguide on the propagation of ligh. Quant. Electron. 25: 927-928 .

11. Vasnetsov M. and Staliunas K. Optical Vortices, Vol. 228 in Horizons of World Physics, Nova Science: Huntington, N.Y. (1999).

12. Mokhun I.I. Introduction to linear singular optics. In Optical Correlation Techniques and Applications, Bellingham: SPIE Press PM168. (2007).

13. Alexeyev CN and Yavorsky MA, 2006. Topological phase evolving from the orbital angular momentum of "coiled" quantum vortices. J. Opt. A: Pure Appl. Opt. 8: 752758

14. Soskin MS, Gorshkov VN, Vasnetsov MV, Malos JT and Heckenberg NR, 1998. Topological charge and angular momentum of light carrying optical vortices. Phys. Rev. A. 56: 4064-4075.

15. Bliokh KYu, 2006. Geometrical optics of beams with vortices: Berry phase and orbital angular momentum Hall effect. Phys. Rev. Lett. 97: 043901 .

16. Alexeyev CN and Yavorsky MA, 2007. Berry's phase for optical vortices in coiled optical fibres. J. Opt. A: Pure Appl. Opt. 9: 6-14.

17. Alexeyev CN, Lapin BA and Yavorsky MA, 2007. Optical vortices and topological phase in strongly anisotropic coiled few-mode optical fibres. J. Opt. Soc. Am. B. 24, 2666-2675.

18. Alexeyev CN and Yavorsky MA, 2007 Propagation of optical vortices in coiled weakly guiding optical fibres. Opt. Spektrosk. 102: 754-759.

19. Alexeyev CN and Yavorsky MA, 2006. Hybridisation of the topological and dynamical phase in coiled optical fibres. J. Opt. A: Pure Appl. Opt. 8: 647-651 .

20. Snyder A.W. and Love J.D. Optical Waveguide Theory. Chapman and Hall: London, New York, (1985).

21. Chen G and Wang Q, 1995. Mode coupling in single-mode helical fibres under perturbation. Opt. Quant. Electron. 27: 1069-74 .

22. Chen G and Wang Q, 1995. Local fields in single-mode helical fibres," Opt. Quant. Electron. 27: 1069-1074.

23. Soh DBS, Nilsson J, Sahu JK and Cooper LJ, 2003. Geometrical factor modification of helical-core fibre radiation loss formula. Opt. Comm. 222: 235-242.

24. Shute MW, Sr., Brown CS and Jarzynski J, 1997. Polarization model for a helically wound optical fibre. J. Opt. Soc. Am. A. 14: 3251-3261 .

Ukr. J. Phys. Opt. 2008, V9, №1 
25. Tsao CYH, 1987. Polarization parameters of plane waves in hybrid birefringent optcal fibres. J. Opt. Soc. Am. A. 4: 1407-1412 .

26. Alexeyev CN, Soskin MS and Volyar AV, 2000. Spin-orbit interaction in a generic vortex field transmitted through an elliptic fibre. Semicond. Phys., Quant. Electron. and Optoelectron. 3: 501-513.

27. Menachem $Z$ and Mond M, 2006. Infrared wave propagation in a helical waveguide with inhomogeneous cross section and application. Electromagn. Waves. 61: 159192.

28. Korn G.A. and Korn T.M., Mathematical handbook for scientists and engineers. McGrawhill: New-York, (1968).

29. V.S. Liberman and B.Ya. Zel'dovich, Spin-orbit interaction of a photon in an inhmogeneous medium. Phys. Rev. A. 45, 5199-5207 (1992).

30. Volyar AV, Zhilaitis VZ and Shvedov VG, 1999. Optical eddies in small-mode fbres:

II. The spin-orbit interaction. Opt. Spektrosk. 86: 593-598.

31. Dooghin AV, Kundikova ND, Liberman VS and Zel'dovich BYa, 1992. Optical Magnus effect. Phys. Rev. A 45: 8204 - 8208 .

32. Bliokh KYu and Bliokh YuP, 2004. Modified geometrical optics of a smoothly inhmogeneous isotropic medium: The anisotropy, Berry phase, and the optical Magnus efect. Phys. Rev. E 70: 026605.

33. Davydov A.S. Quantum mechanics. Pergamon: Oxford, (1976).

34. Alexeyev CN and Yavorsky MA, 2004. Optical vortices and the higher order modes of twisted strongly elliptical optical fibres. J. Opt. A: Pure Appl. Opt. 6: 824-832.

35. Alexeyev CN and Yavorsky MA, 2007. Pancharatnam's phase induced by spin-orbit interaction in weakly guiding twisted elliptical fibres. Ukr. J. Phys. Opt. 8: 1-12 .

36. Pancharatnam S., Collected works of S. Pancharatnam. Oxford: University Press, (1975).

37. Berry MV, 1987. The adiabatic phase and Pancharatnam's phase for polarized light. J. Mod. Opt. 34: 1401-07 . 